\title{
SENSIBILIDAD MICROBIANA DE ESCHERICHIA COLI EN BACTERIURIAS EN EL ÁREA SANITARIA DEL BIERZO EN EL AÑO 2003
}

\author{
J.M. SÁNCHEZ MERINO, C. GUILLÁN MAQUIEIRA*, C. FUSTER FOZ**, \\ R. LÓPEZ MEDRANO**, M. JIMÉNEZ RODRÍGUEZ***, J. GARCÍA ALONSO
}

Servicios de Urología, *Ginecología, **Microbiología y ***Medicina Preventiva. Hospital del Bierzo. Ponferrada. León.

Actas Urol Esp. 28 (8): 588-593, 2004

\section{RESUMEN}

SENSIBILIDAD MICROBIANA DE ESCHERICHIA COLI EN BACTERIURIAS EN EL ÁREA SANITARIA DEL BIERZO EN EL AÑO 2003

OBJETIVO: Evaluar la sensibilidad a varios antibióticos en cepas de Escherichia coli aisladas en muestras de orina de pacientes hospitalarios y extrahospitalarios.

MATERIAL Y MÉTODOS: Se analizaron los urocultivos positivos para Escherichia coli obtenidos de muestras de pacientes hospitalizados y atendidos en consultas externas hospitalarias y atención primaria. Se determinó la sensibilidad de este germen a diecinueve antibióticos de uso hospitalario y/o extrahospitalario.

RESULTADOS: Según el origen hospitalario, consultas externas hospitalarias y atención primaria, respectivamente, la sensibilidad in vitro ha sido la siguiente: imipenem (100\%, 100\% y 100\%; $\mathrm{p}=\mathrm{NS})$, amikacina (100\%, 100\% y 99,7\%; $\mathrm{p}=\mathrm{NS})$, fosfomicina (98,6\%, 98,4\% y 99,6\%; p=NS), cefepima (96\%, 96,9\% y 98,3\%; p=NS), piperacilina-tazobactam $(96 \%$, 95,3\% y $96,6 \%$; p=NS), aztreonam (93,5\%, 94,7\% y 97,7\%; p<0,001), ceftazidima (93,5\%, 94,3\% y 97,8\%; p<0,001), cefotaxima (93,1\%, 95\% y 97,7\%; p<0,001), cefixima (92,7\%, 94,6\% y 96,7\%; p<0,05), nitrofurantoína (92\%, 94,7\% y 94,7\%; p=NS), cefuroxima $(88,4 \%, 93,1 \%$ y 95\%; p<0,001), amoxicilina-clavulánico $(87,7 \%, 88,7 \%$ y 93,8\%; p<0,001), tobramicina $(87 \%$, 93,7\% y $93,8 \%$; p<0,001), gentamicina $(85,9 \%, 92,8 \%$ y $93 \%$; p<0,001), cefazolina $(84,4 \%, 88,7 \%$ y $91,6 \%$; p<0,01), ciprofloxacino $(63,8 \%, 71,4 \%, 78,4 \%$; p <0,001), norfloxacino $(63 \%, 70,8$ y 78,2\%; p<0,001), cotrimoxazol $(65,2 \%, 68,6 \%$ y $74,9 \%$; p<0,01) y ampicilina (35,5\%, 42,5\% y 47,8\%; p<0,01). (NS= No significativo).

CONCLUSIONES: El tratamiento de las infecciones del tracto urinario frecuentemente se inicia de forma empírica. Por este motivo resulta esencial conocer los patrones de sensibilidad de las bacterias potencialmente causantes del cuadro.

PALABRAS CLAVE: Infección del tracto urinario. Sensibilidad microbiana. Infección hospitalaria. Infección extrahospitalaria.

\section{ABSTRACT}

\section{ANTIMICROBIAL SUSCEPTIBILITY OF ESCHERICHIA COLI ISOLATED FROM BACTERIURIAS IN BIERZO HEALTH} AREA DURING 2003

OBJECTIVE: Sensitivity to antibiotics of Escherichia coli isolated from intrahospital and extrahospital bacteriurias is analized.

MATERIAL AND METHODS: Sensitivity to nineteen antibiotics used in the clinical practise was determined in strains of Escherichia coli isolated from intrahospital and extrahospital bacteriurias.

RESULTS: The prevalence of in-vitro susceptibilities to antibiotics were (hospital, state health service clinic, health center-primary-care): imipenem (100\%-100\%-100\%; p=NS), amikacin (100\%-100\%-99.7\%; p=NS), fosfomicyn (98.6\%98.4\%-99.6\%; p=NS), cefepime (96\%-96.9\%-98.3\%; p=NS), piperacillin-tazobactam (96\%-95.3\%-96.6\%; p=NS), aztreonam (93.5\%-94.7\%-97.7\%; p<0.001), ceftazidime (93.5\%-94.3\%-97.8\%; p<0.001), cefotaxime (93.1\%-95\%-97.7\%; $\mathrm{p}<0.001)$, cefixime (92.7\%-94.6\%-96.7\%; p<0.05), nitrofurantoin (92\%-94.7\%-94.7\%; $\mathrm{p}=\mathrm{NS})$, cefuroxime $(88.4 \%-93.1 \%$ 95\%; p<0.001), amoxicillin-clavulanic (87.7\%-88.7\%-93.8\%; p<0.001), tobramicyn (87\%-93.7\%-93.8\%; p<0.001), gentamcin (85.9\%-92.8\%-93\%; p<0.001), cefazolin (84.4\%-88.7\%-91.6\%; p<0.01), ciprofloxacin $(63.8 \%-71.4 \%-78.4 \%$; $\mathrm{p}<0.001)$, norfloxacin (63\%-70.8\%-78.2\%; p<0.001), cotrimoxazole $(65.2 \%-68.6 \%-74.9 \% ; \mathrm{p}<0.01)$ and ampicillin (35.5\%-42.5\%-47.8\%; p<0.01). ( ${ }^{*} N S=$ No significant differences).

CONCLUSIONS: The knowledge of the sensitivity of uropathogens to antimicrobians can allow us to initiate empirical therapy of urinary tract infections.

KEY WORDS: Urinary tract infection. Antibiotic susceptibility. Intrahospital infection. Community-acquired infection. 
$E_{\text {i }}$ scherichia coli es la causa más común de infección del tracto urinario, tanto en pacientes hospitalizados como ambulatorios. Así, se ha aislado hasta en un 50\% de los pacientes hospitalizados y el 63\% de los pacientes diagnosticados de infección urinaria de origen ambulatorio $^{1}$. En atención primaria puede suponer entre el 63 y el $82 \%$ de los aislamientos urinarios ${ }^{2,3}$.

El tratamiento de las infecciones del tracto urinario frecuentemente se inicia de forma empírica. Por este motivo y dada la prevalencia creciente de resistencias resulta esencial conocer los patrones de sensibilidad de las bacterias potencialmente causantes del cuadro.

El propósito de este estudio es evaluar la sensibilidad a varios antibióticos, de administración oral y/o parenteral, en cepas de Escherichia coli aisladas en muestras de orina de pacientes del Área Sanitaria del Hospital del Bierzo (León) en el año 2003 y detectar las variaciones de sensibilidad según el ámbito de referencia (hospitalario, ambulatorio y atención primaria).

\section{MATERIAL Y METODOS}

Se realizó un estudio retrospectivo de los urocultivos positivos para Escherichia coli obtenidos de muestras enviadas al Laboratorio de Microbiología del Área de Salud del Bierzo (150.000 habitantes) de pacientes atendidos en tres ámbitos distintos: hospitalización, consultas externas hospitalarias y atención primaria. Los urocultivos analizados corresponden a la totalidad de los positivos para Escherichia coli, procedentes de pacientes con diagnóstico de sospecha de infección urinaria y/o control postratamiento. Resultaron positivos para Escherichia coli 276 urocultivos hospitalarios, 318 procedentes de consultas externas hospitalarias y 901 de atención primaria. No se realizó distinción según especialidades hospitalarias. Tampoco se discriminó según la especialidad de origen de consultas externas hospitalarias.

Se estudió la sensibilidad in vitro de Escherichia coli a diecinueve antibióticos, de administración oral y/o parenteral (Tabla 1). Algunos de los de administración exclusivamente parenteral se pueden pautar con receta médica habitual (aztreonam $500 \mathrm{mg}$ y $1 \mathrm{~g}$, cefazolina $500 \mathrm{mg}$ y $1 \mathrm{~g}$ ); otros son de diagnóstico hospitalario (imipenem 500 mg,
Tabla 1

Antibióticos utilizados para el estudio de sensibilidad in vitro de Escherichia coli

\begin{tabular}{|c|c|c|}
\hline & Aminopenicilinas & $\begin{array}{l}\text { Ampicilinab }^{b} \\
\text { Amoxicilina- } \\
\text { clavulánico }^{b}\end{array}$ \\
\hline & Monobactámicos & Aztreonamc \\
\hline & Carbapenemes & Imipenem $^{c}$ \\
\hline & Ureidopenicilinas & $\begin{array}{l}\text { Piperacilina- } \\
\text { tazobactam }^{c}\end{array}$ \\
\hline \multirow[t]{4}{*}{$\beta$-Lactámicos } & $\begin{array}{l}\text { Cefalosporinas de } \\
1^{\underline{a}} \text { generación }\end{array}$ & Cefazolinac \\
\hline & $\begin{array}{l}\text { Cefalosporinas de } \\
2^{\mathrm{a}} \text { generación }\end{array}$ & Cefuroxima ${ }^{b}$ \\
\hline & $\begin{array}{l}\text { Cefalosporinas de } \\
3^{a} \text { generación }\end{array}$ & $\begin{array}{l}\text { Cefixima }^{a} \\
\text { Cefotaxima }^{c} \\
\text { Ceftazidimac }^{c}\end{array}$ \\
\hline & $\begin{array}{l}\text { Cefalosporinas de } \\
4^{\text {a }} \text { generación }\end{array}$ & Cefepimac \\
\hline Quinolonas & \multicolumn{2}{|c|}{$\begin{array}{l}\text { Norfloxacino }^{\mathrm{a}} \\
\text { Ciprofloxacino }^{\mathrm{b}}\end{array}$} \\
\hline Aminoglucósidos & \multicolumn{2}{|c|}{$\begin{array}{l}\text { Amikacinac } \\
\text { Gentamicinac } \\
\text { Tobramicinac }\end{array}$} \\
\hline \multicolumn{3}{|c|}{ Fosfomicina ${ }^{b}$} \\
\hline \multicolumn{3}{|c|}{ Nitrofurantoína ${ }^{a}$} \\
\hline
\end{tabular}

En cursiva: Inhibidores de las $\beta$-lactamasas.

a: Administración oral. b: Administración oral y parenteral.

c: Administración parenteral.

cefotaxima $250 \mathrm{mg}, 500 \mathrm{mg} \mathrm{y} 1 \mathrm{~g}$, ceftazidima $500 \mathrm{mg}$ y $1 \mathrm{gr}$, cefepima $500 \mathrm{mg}$ y $1 \mathrm{~g}$ ) o de uso hospitalario (aztreonam 2 g, imipenem 250 y 500 mg i.v., piperacilina-tazobactam 2/0,25 y 4/0,5 $\mathrm{g}$, cefazolina $2 \mathrm{~g}$, cefotaxima $2 \mathrm{~g}$, ceftazidima $\lg \mathrm{y}$ $2 \mathrm{~g}$, cefepima $2 \mathrm{~g}$ ).

Se determinaron las concentraciones mínimas inhibitorias mediante el sistema MicroScan ${ }^{\circledR}$ (Dade Behring). En la interpretación de la sensibilidad se aplicaron los criterios del NCCLS (National Committe for Clinical Laboratory Standards) de $2003^{4}$ (Tabla 2).

Se aplicó la prueba de chi cuadrado para valorar si existieron diferencias significativas $(\mathrm{p}<0,05)$ de sensibilidad de Escherichia coli a los antibióticos referidos entre las distintas áreas estudiadas (hospitalización, consultas externas hospitalarias y atención primaria). En la comparación se excluyeron las cepas de sensibilidad intermedia. 
Tabla 2

Valores de la concentración mínima inhibitoria para la interpretación de la sensibilidad, según el NCCLS ${ }^{4}$

\begin{tabular}{ll}
\hline Antimicrobiano & Sensible \\
Amikacina & $\leq 16$ \\
Amoxicilina-clavulánico & $\leq 8 / 4$ \\
Ampicilina & $\leq 8$ \\
Aztreonam & $\leq 8$ \\
Cefazolina & $\leq 8$ \\
Cefepima & $\leq 8$ \\
Cefixima & $\leq 1$ \\
Cefotaxima & $\leq 8$ \\
Ceftazidima & $\leq 8$ \\
Cefuroxima & $\leq 8$ \\
Ciprofloxacino & $\leq 1$ \\
Cotrimoxazol & $\leq 2 / 38$ \\
Fosfomicina & $\leq 64$ \\
Gentamicina & $\leq 4$ \\
Imipenem & $\leq 4$ \\
Nitrofurantoína & $\leq 32$ \\
Norfloxacino & $\leq 4$ \\
Piperacilina-tazobactam & $\leq 16 / 4$ \\
Tobramicina & $\leq 4$ \\
\hline
\end{tabular}

Valores en expresados en $\mu \mathrm{g} / \mathrm{mL}$

\section{RESULTADOS}

Se obtuvo el crecimiento de Escherichia coli en el 49,7\%, 53,2\% y 59,8\% de los urocultivos positivos enviados desde el hospital, consultas externas hospitalarias y atención primaria, respectivamente. La Tabla 3 muestra los gérmenes más frecuentemente causantes de bacteriurias en nuestro medio según el origen de la muestra.

La Tabla 4 resume la comparación global de la sensibilidad in vitro de Escherichia coli según la procedencia. La mejor actividad, cualquiera que fuese el origen, la presentaron el imipenem y la amikacina. Por otra parte, Escherichia coli también mantuvo una excelente sensibilidad a fosfomicina, sin diferencias estadísticamente significativas de sensibilidad según el nivel de procedencia. Cefepima y piperacilina-tazobactam, independientemente del origen del urocultivo, presentaron una buena actividad, por encima del 95\% de cepas.
Aztreonam, ceftazidima, cefotaxima, cefixima y nitrofurantoína mostraron una actividad por encima del 92\%. No obstante, los tres primeros obtuvieron mejor actividad en cepas de atención primaria que en cepas de hospital, con una diferencia estadísticamente significativa $(p<0,01$ para aztreonam y $\mathrm{p}<0,001$ para ceftazidima $\mathrm{y}$ cefotaxima). Lo mismo ocurrió con respecto a los urocultivos procedentes de consultas externas $(\mathrm{p}<0,05$ para aztreonam y cefotaxima y $\mathrm{p}<0,01$ para ceftazidima). Sin embargo, no se observaron diferencias estadísticamente significativas entre los ámbitos hospitalario y de consultas externas. Sólo se observaron diferencias estadísticamente significativas de sensibilidad para cefixima entre el hospital y atención primaria $(\mathrm{p}<0,01)$. La nitrofurantoína mantuvo similar actividad, independientemente del origen.

Se pueden considerar de actividad aceptable a nivel extrahospitalario cefuroxima, amoxicilinaclavulánico, tobramicina, gentamicina y cefazolina, aunque esta varía según se trate de muestras de atención primaria o consultas externas hospitalarias. Cefuroxima mantiene una mayor actividad, estadísticamente significativa, en cepas procedentes de atención primaria con respecto al hospital ( $\mathrm{p}<0,001)$. Sin embargo, tal diferencia no se observa cuando se comparan las cepas del hospital con respecto a las de consultas externas hospitalarias y de éstas con las de atención primaria. Amoxicilina-clavulánico presentó una mayor actividad en atención primaria que en cualquiera de los otros ámbitos $(\mathrm{p}<0,01)$, pero no existieron diferencias estadísticamente significativas entre las consultas externas y el hospital. La actividad para tobramicina y gentamicina fue mayor en atención primaria $(\mathrm{p}<0,001)$ y consultas externas $(p<0,01)$ que en el hospital. Sin embargo, no existieron diferencias a nivel extrahospitalario. Sólo se observaron diferencias significativas de actividad para cefazolina entre en hospital y atención primaria $(\mathrm{p}<0,001)$.

Ciprofloxacino, norfloxacino y cotrimoxazol presentaron diferencias estadísticamente significativas en la comparación del hospital con atención primaria ( $\mathrm{p}<0,001$ para las fluoroquinolonas y $\mathrm{p}<0,01$ para cotrimoxazol) y consultas externas con atención primaria ( $\mathrm{p}<0,05$ para ciprofloxacino y cotrimoxazol y $\mathrm{p}<0,01$ para norfloxacino). 
Tabla 3

Distribución de los gérmenes más frecuentes causantes de bacteriurias hospitalarias y extrahospitalarias

\begin{tabular}{|c|c|c|c|c|c|c|c|c|}
\hline \multicolumn{3}{|c|}{$\begin{array}{l}\text { Hospital } \\
\text { N = 555 }\end{array}$} & \multicolumn{3}{|c|}{$\begin{array}{l}\text { Consultas externas } \\
\qquad \mathbf{N}=598\end{array}$} & \multicolumn{3}{|c|}{$\begin{array}{l}\text { Atención primaria } \\
\qquad N=1502\end{array}$} \\
\hline Germen & $\mathbf{N}$ & $\%$ & Germen & $\mathbf{N}$ & $\%$ & Germen & $\mathbf{N}$ & $\%$ \\
\hline Escherichia coli & 276 & 49,7 & Escherichia coli & 318 & 53,2 & Escherichia coli & 901 & 59,8 \\
\hline $\begin{array}{l}\text { Enterococcus } \\
\text { faecalis }\end{array}$ & 88 & 15,9 & $\begin{array}{l}\text { Enterococcus } \\
\text { faecalis }\end{array}$ & 98 & 16,4 & $\begin{array}{l}\text { Enterococcus } \\
\text { faecalis }\end{array}$ & 178 & 11,8 \\
\hline Candida albicans & 51 & 9,2 & $\begin{array}{l}\text { Streptococcus } \\
\text { agalactiae }\end{array}$ & 35 & 5,9 & Proteus mirabilis & 96 & 6,4 \\
\hline $\begin{array}{l}\text { Pseudomona } \\
\text { aeruginosa }\end{array}$ & 24 & 4,3 & $\begin{array}{l}\text { Klebsiella } \\
\text { pneumoniae }\end{array}$ & 26 & 4,3 & $\begin{array}{l}\text { Streptococcus } \\
\text { agalactiae }\end{array}$ & 73 & 4,8 \\
\hline Proteus mirabilis & 18 & 3,2 & Proteus mirabilis & 26 & 4,3 & $\begin{array}{l}\text { Klebsiella } \\
\text { pneumoniae }\end{array}$ & 53 & 3,5 \\
\hline $\begin{array}{l}\text { Klebsiella } \\
\text { pneumoniae }\end{array}$ & 16 & 2,9 & $\begin{array}{l}\text { Pseudomona } \\
\text { aeruginosa }\end{array}$ & 19 & 3,2 & $\begin{array}{l}\text { Pseudomona } \\
\text { aeruginosa }\end{array}$ & 29 & 1,9 \\
\hline $\begin{array}{l}\text { Staphylococcus } \\
\text { aureus }\end{array}$ & 14 & 2,5 & Klebsiella oxytoca & 13 & 2,2 & $\begin{array}{l}\text { Morganella } \\
\text { morganii }\end{array}$ & 28 & 1,9 \\
\hline $\begin{array}{l}\text { Morganella } \\
\text { morganii }\end{array}$ & 10 & 1,8 & $\begin{array}{l}\text { Staphylococcus } \\
\text { aureus }\end{array}$ & 10 & 1,7 & Klebsiella oxytoca & 24 & 1,6 \\
\hline $\begin{array}{l}\text { Enterobacter } \\
\text { cloaceae }\end{array}$ & $\begin{array}{l}8 \\
8\end{array}$ & $\begin{array}{l}1,4 \\
1,4\end{array}$ & $\begin{array}{l}\text { Citrobacter } \\
\text { freundii }\end{array}$ & 8 & 1,3 & $\begin{array}{l}\text { Enterobacter } \\
\text { cloaceae }\end{array}$ & 21 & 1,4 \\
\hline $\begin{array}{l}\text { Klebsiella } \\
\text { oxytoca }\end{array}$ & 8 & 1,4 & $\begin{array}{l}\text { Enterobacter } \\
\text { cloaceae }\end{array}$ & 8 & 1,3 & $\begin{array}{l}\text { Staphylococcus } \\
\text { aureus }\end{array}$ & 17 & 1,1 \\
\hline Otros & 42 & 7,6 & Otros & 37 & 6,2 & Otros & 86 & 5,7 \\
\hline
\end{tabular}

$\mathrm{N}$ : número de urocultivos positivos en el año 2003.

Por último, para ampicilina sólo se observaron diferencias estadísticamente significativas en la comparación del hospital con atención primaria $(\mathrm{p}<0,001)$.

\section{DISCUSIÓN}

El tratamiento empírico de la infección del tracto urinario es una práctica habitual. Por lo tanto, el conocimiento del patrón de sensibilidad antibiótica de los gérmenes más comunes es fundamental para orientar adecuadamente el tratamiento ${ }^{5}$.

Escherichia coli ha sido el germen más frecuentemente aislado en muestras de orina de pacientes atendidos en el Área de Salud del Bierzo. La distribución de los microorganismos en el medio hospitalario y extrahospitalario en trabajos recientes en nuestro país es muy semejante ${ }^{1,2,6}$.

En nuestro estudio el imipenem y la amikacina mostraron una excelente actividad in vitro frente a Escherichia coli, de casi el 100\%, en el conjunto de las muestras. El imipenem es un antibiótico $\beta$-lactámico de amplio espectro para microorganismos grampositivos y gramnegativos aerobios y anaerobios ${ }^{5}$. Se administra asociado a la cilastatina, un inhibidor enzimático que impide su destrucción en el riñón.

Los aminoglucósidos no son de uso habitual en las infecciones del tracto urinario no complicadas, aunque son eficaces en monodosis en el $90 \%$ de las mismas ${ }^{5}$. Por otra parte, en las infecciones del tracto urinario complicadas la asociación con un $\beta$-lactámico es altamente eficaz ${ }^{4,5}$. Dentro de este grupo de antibióticos, la amikacina es activa frente a casi todas las cepas de Escherichia coli resistentes a otros aminoglucósidos más utilizados ${ }^{5}$. Esta diferencia de sensibilidad radica en su distinta afectación por los enzimas inactivadores de aminoglucósidos ${ }^{4}$.

La fosfomicina presentó en nuestro estudio una excelente actividad in vitro frente a Escherichia coli. Este aspecto, junto con la infrecuencia de efectos adversos y las concentraciones urinarias alcanzadas, convierten a la fosfomicina trometamol en una alternativa a tener en cuenta en el tratamiento de la infección urinaria no complicada ${ }^{7}$. 
Tabla 4

Comparación global de la sensibilidad microbiana de Escherichia coli en urocultivos del hospital, consultas externas hospitalarias y atención primaria

\begin{tabular}{|c|c|c|c|c|c|c|c|c|c|c|}
\hline & \multirow[b]{2}{*}{$\mathbf{N}$} & \multicolumn{2}{|l|}{ Hospital } & \multicolumn{3}{|c|}{ Consultas externas } & \multicolumn{3}{|c|}{ Atención Primaria } & \multirow[b]{2}{*}{$\mathbf{p}$} \\
\hline & & Sensibles & $\%$ & $\mathbf{N}$ & Sensibles & $\%$ & $\mathbf{N}$ & Sensibles & $\%$ & \\
\hline Imipenem & 276 & 276 & 100 & 318 & 318 & 100 & 901 & 901 & 100 & NS \\
\hline Amikacina & 276 & 276 & 100 & 318 & 318 & 100 & 901 & 898 & 99,7 & NS \\
\hline Fosfomicina & 276 & 272 & 98,6 & 318 & 313 & 98,4 & 901 & 897 & 99,6 & NS \\
\hline Cefepima & 276 & 265 & 96 & 318 & 308 & 96,9 & 901 & 886 & 98,3 & NS \\
\hline Piper-tazobact $^{\mathbf{1}}$ & 276 & 265 & 96 & 318 & 303 & 95,3 & 901 & 870 & 96,6 & NS \\
\hline Aztreonam & 276 & 258 & 93,5 & 318 & 301 & 94,7 & 901 & 880 & 97,7 & $\mathrm{p}<0,001$ \\
\hline Ceftazidima & 276 & 258 & 93,5 & 318 & 300 & 94,3 & 901 & 881 & 97,8 & $\mathrm{p}<0,001$ \\
\hline Cefotaxima & 276 & 257 & 93,1 & 318 & 302 & 95 & 901 & 880 & 97,7 & $\mathrm{p}<0,001$ \\
\hline Cefixima & 274 & 254 & 92,7 & 317 & 300 & 94,6 & 900 & 870 & 96,7 & $\mathrm{p}<0,05$ \\
\hline Nitrofurantoína & 276 & 254 & 92 & 318 & 301 & 94,7 & 901 & 853 & 94,7 & NS \\
\hline Cefuroxima & 276 & 244 & 88,4 & 318 & 296 & 93,1 & 901 & 856 & 95 & $\mathrm{p}<0,001$ \\
\hline Amox-clavulán ${ }^{2}$ & 276 & 242 & 87,7 & 318 & 282 & 88,7 & 901 & 845 & 93,8 & $\mathrm{p}<0,001$ \\
\hline Tobramicina & 276 & 240 & 87 & 318 & 298 & 93,7 & 901 & 845 & 93,8 & $\mathrm{p}<0,001$ \\
\hline Gentamicina & 276 & 237 & 85,9 & 318 & 295 & 92,8 & 901 & 838 & 93 & $\mathrm{p}<0,001$ \\
\hline Cefazolina & 276 & 233 & 84,4 & 318 & 282 & 88,7 & 901 & 825 & 91,6 & $\mathrm{p}<0,01$ \\
\hline Ciprofloxacino & 276 & 176 & 63,8 & 318 & 227 & 71,4 & 901 & 706 & 78,4 & $\mathrm{p}<0,001$ \\
\hline Norfloxacino & 276 & 174 & 63 & 318 & 225 & 70,8 & 901 & 705 & 78,2 & $\mathrm{p}<0,001$ \\
\hline Cotrimoxazol & 276 & 180 & 65,2 & 318 & 218 & 68,6 & 901 & 675 & 74,9 & $\mathrm{p}<0,01$ \\
\hline Ampicilina & 276 & 98 & 35,5 & 318 & 135 & 42,5 & 901 & 431 & 47,8 & $\mathrm{p}<0,01$ \\
\hline
\end{tabular}

1: Piperacilina-tazobactam. ${ }^{2}$ : Amoxicilina-clavulánico. $\mathrm{N}$ : número de urocultivos en los que se probó la sensibilidad al antibiótico. NS: no significativo.

Las comparaciones del hospital con atención primaria, hospital con consultas externas y consultas externas con atención primaria se explican en el texto.

Algunos de los antibióticos estudiados son de uso hospitalario.

Cefepima es una cefalosporina de $4^{\text {a }}$ generación más estable a las $\beta$-lactamasas que el resto de las cefalosporinas, lo que la hace una opción válida frente a bacilos gramnegativos aerobios resistentes a aquellas ${ }^{5}$. En nuestro estudio mantuvo una buena actividad frente a Escherichia coli, independientemente del origen de la muestra. Similar comportamiento ha presentado la piperacilina-tazobactam, combinación que incluye una ureidopenicilina (piperacilina) y un segundo agente (tazobactam) que tiene mínima actividad antibacteriana, pero funciona como un inhibidor de algunas $\beta$-lactamasas ${ }^{4}$.

El aztreonam, antibiótico $\beta$-lactámico del grupo de los monobactames, ha presentado una mayor sensibilidad a nivel extrahospitalario en nuestra serie, en probable relación con una menor utilización fuera del hospital.
Las cefalosporinas de $3^{\text {a }}$ generación ceftazidima, cefotaxima y cefixima mantuvieron una aceptable actividad frente a Escherichia coli en nuestro estudio. En general, son activas frente a gramnegativos nosocomiales y enterobacterias ${ }^{5}$.

La nitrofurantoína, la cefuroxima y la amoxicilina-clavulánico presentaron una actividad suficientemente elevada para considerarlas adecuadas para el tratamiento empírico de la infección urinaria extrahospitalaria, pero no así a nivel intrahospitalario. En general, la sensibilidad mostrada por Escherichia coli a estos antibióticos en nuestro medio está en consonancia con la reflejada en la literatura ${ }^{2,8}$. La nitrofurantoína, usada específicamente para tratar las infecciones urinarias adquiridas en la comunidad, todavía presenta unos niveles sorprendentemente bajos de resistencias, a pesar de los años de uso. 
El grupo de las fluoroquinolonas incluye un número de agentes relacionados estrechamente que funcionan primariamente por inhibición de la actividad de la DNA-girasa y de la topoisomerasa IV de muchas bacterias grampositivas y gramnegativas ${ }^{4}$. Su amplio espectro de acción, potencia bactericida, propiedades farmacocinéticas (buena biodisponibilidad oral, baja unión a proteínas plasmáticas y eliminación renal) y su buena tolerancia las han convertido en uno de los grupos de antibióticos de más amplia aceptación ${ }^{1}$. Sin embargo, en España las cifras de resistencias a las quinolonas se han disparado debido a su uso masivo en la práctica clínica ${ }^{8}$. En nuestro medio concreto la tasa de resistencias oscila entre 22 y $37 \%$, dependiendo del ámbito de estudio.

Escherichia coli ha mostrado una baja sensibilidad a cotrimoxazol y ampicilina, que por otro lado permanece estable en España con el paso de los años ${ }^{3}$.

El patrón de sensibilidad in vitro obtenido para Escherichia coli en el área de salud del Bierzo permitiría aplicar las pautas de tratamiento empírico de la infección urinaria recomendadas habitualmente en España ${ }^{9}$, pero desaconsejando el uso de las fluoroquinolonas ${ }^{10}$. Así, en nuestro medio serían adecuados para el tratamiento empírico, según el cuadro infeccioso, los siguientes antimicrobianos: a) cistitis simple: fosfomicina trometamol, cefalosporina oral de $2^{\mathrm{a}}$ o $3^{\mathrm{a}}$ generación, amoxicilina-clavulánico o nitrofurantoína; b) cistitis complicada: cefalosporina oral de $2^{\underline{a}}$ o $3^{\underline{a}}$ generación, con una duración mayor del tratamiento que en el cuadro anterior; c) pielonefritis aguda sin criterios de ingreso hospitalario: cefalosporina oral de $3^{\text {a }}$ generación, cefalosporina intramuscular de $2^{\underline{a}}$ o $3^{\text {a }}$ generación o un aminoglucósido intramuscular en dosis única diaria; d) pielonefritis aguda con criterios de ingreso hospitalario pero sin factores de riesgo de infección por microorganismos multirresistentes y hemodinámicamente estable: cefalosporina de $3^{\underline{a}}$ generación, aztreonam o aminoglucósido en dosis única diaria. Si existe riesgo de infección por gérmenes multirresistentes y/o inestabilidad hemodinámica: cefalosporina antipseudomónica (ceftazidima, cefepima) o aztreonam asociados a ampicilina, o bien monoterapia con un carbapenem o piperacilina-tazobactam. En caso de shock séptico se debe añadir un aminoglucósido a cualquiera de las pautas anteriores ${ }^{9}$.
En general, las recomendaciones terapéuticas difieren en Estados Unidos, país donde las resistencias a fluoroquinolonas y cotrimoxazol son sensiblemente inferiores ${ }^{11,12}$.

Finalmente, aunque no se tuvo en cuenta las características clínicas de los pacientes, parece razonable utilizar los datos proporcionados por los laboratorios de microbiología como orientación para el tratamiento empírico de las infecciones urinarias. No obstante, la mejor aproximación se obtendría estratificando los resultados por edad, sexo y tipo de patología.

\section{REFERENCIAS}

1. Queipo JA, Budía A, Jiménez MJ et al. Evolución de la resistencia microbiana a fluorquinolonas en un hospital terciario. Actas Urol Esp 2000;24(5):381-387.

2. Queipo JA, Giménez MJ, Diosdado N, Gómez-Ferrer A, Gobernado M, Jiménez JF. Cistitis aguda en la mujer. Sensibilidad microbiana actual en nuestro medio. Actas Urol Esp 2001;25(8): 567-572.

3. Sánchez Merino JM, Guillán C, Fuster C, Madrid FJ, Jiménez M, García J. Sensibilidad microbiana de Escherichia coli en infecciones urinarias extrahospitalarias. Actas Urol Esp 2003;27(10): 783-787.

4. NCCLS. Methods for Dilution Antimicrobial Susceptibility Tests for Bacteria That Grow Aerobically; Approved Standard - Sixth Edition. NCCLS document M7-A6 (ISBN 1-56238-486-4). NCCLS, 940 West Valley Road, Suite 1400; Wayne, Pennsylvania 19087 1898 USA, 2003.

5. Navío S, Gómez V, Clemente L. Epidemiología y bases farmacológicas de las infecciones del tracto urinario. En: Navío S, editor. Patología urológica infecciosa. Madrid: Aula Médica, Ediciones; 1999:1-13.

6. Kahlmeter G. The ECO-SENS Project: a prospective, multinational, multicentre epidemiological survey of the prevalence and antimicrobial susceptibility of urinary tract pathogens-interim report. J Antimicrob Chemother 2000;46(Suppl S1):15-22.

7. Garcia-Rodríguez JA, Trujillano I, Baquero F et al. In Vitro activity of fosfomycin trometamol against pathogens from urinary tract infections: a Spanish multicenter study. J Chemother 1997;9(6): 394-402.

8. Jiménez JF, Broseta E, Gobernado M. Infección urinaria. Actas Urol Esp 2002;26(7):563-573.

9. Mensa J, Gatell JM, Jiménez MT, Prats G, Dominguez-Gil A, editores. Guía de Terapéutica Antimicrobiana. 14 ${ }^{\mathrm{a}}$ ed. Barcelona: Masson; 2004.

10. Mauri E, Garau J. Pielonefritis aguda. En: Carballido J, Gobernado M, editores. Patología infecciosa en Urología. Madrid: Ediciones Ergon, S.A.; 1998:305-309.

11. Krieger JN. Urinary tract infections: What's new?. J Urol 2002; 168:2351-2358.

12. Gilbert DN, Moellering RC, Sande MA, editors. The sanford guide to antimicrobial therapy 2003. 33 ${ }^{\mathrm{RD}}$ Edition. Hyde Park (USA): Antimicrobial Therapy Inc; 2003.

Dr. J.M. Sánchez Merino

C/ Francisco González, 25

24400 Ponferrada (León)

(Trabajo recibido el 19 abril de 2004) 